\title{
Basic research and applied research
}

Scientists are under pressure today to demonstrate the practical outcomes (in terms of benefit to society) of the research they do, which is almost entirely funded by public funds provided by the government. This pressure has led to scientists as well as funding agencies favouring applied research over basic research. It has been argued that a country such as Sri Lanka cannot afford the luxury of supporting basic research.

In this context, it is worthwhile to examine the natures of basic and applied research and their impact on each other and society. The classification of research into basic and applied does not denote a fundamental difference in the type of activity that scientists are involved in, but in their goals. In brief, the goal of basic research is to understand while that of applied research is to use.

The strongest argument for funding basic research is that yesterday's basic research is tomorrow's applied research. The proponents of basic research as an essential activity for technological development instinctively appeal to a linear model, traversing from basic research through applied research to development research and technology/ products. In this model, it is the knowledge generated by basic research that enables the applied researcher to explore the feasibility of converting the potential inherent in that knowledge towards practical outcomes.
The linear model has been criticised as being inconsistent with actual history of science and technology, and on the observation that there is a continuous interplay between new technology and basic research. An instance is the developments in thermodynamics that came after the invention of the steam engine. The development of the field of microbiology spurred by Louis Pasteur's interest in the practical problems of fermentation and spoilage is cited as a prime example of research driven by the desire of a practical outcome leading to fundamental developments in science.

In order to increase the social relevance of basic research, funding agencies of scientific research tend to preferably fund mission-oriented basic research, where the broad research area is determined by the agency, but the researcher is left free to pursue research without targeting a practical outcome. The Journal of the National Science Foundation publishes high quality papers reporting results from both basic and applied research. However, papers presenting results of development research which mainly present empirical data gathered by systematic variation of experimental parameters aimed at product optimisation, without generating new knowledge or understanding are generally considered outside the scope of the Journal.

Ajit Abeysekera 